\title{
CAUSES OF CERVICAL LYMPHADENOPATHY - A CYTOLOGIC STUDY
}

C. Nirmala ${ }^{1}$, Dayananda. S. Biligi ${ }^{2}$, Radha ${ }^{3}$

\section{HOW TO CITE THIS ARTICLE:}

C. Nirmala, Dayananda. S. Biligi, Radha. "Causes of Cervical Lymphadenopathy - A Cytologic Study".Journal of Evolution of Medical and Dental Sciences 2014; Vol. 3, Issue 02, January 13; Page: 379-385,

DOI:10.14260/jemds/2014/1837

ABSTRACT: Cervical lymphadenopathy is a common clinical presentation in various departments of clinical practice. This study was done to know the prevalence of causes responsible for cervical lymphadenopathy, distribution of lesions among the age \& sex groups and to emphasize the role of fine needle aspiration cytology (FNAC) in etiologic workup in cervical lymphadenopathy. 420 patients with cervical lymphadenopathy were assessed clinically, by laboratory and by FNAC study over a period of one year. The results concluded that tuberculosis is the most common cause of cervical lymphadenopathy in the south Asian population followed by reactive lymphadenitis and metastatic deposits. Reactive lymphadenitis is the commonest cause of cervical lymph node enlargement in pediatric age group, infective in 11-40 yrs. age group including tuberculous and suppurative lymphadenitis, neoplastic lesions in more than 40 age group including metastatic disease and nonhodgkins lymphomas. FNAC is an important diagnostic modality for the etiologic workup in significant cervical lymphadenopathy and is almost as sensitive and specific as excision lymph node biopsy when an adequate aspirate is examined by expert eyes.

KEY WORDS: Cervical lymphadenopathy, FNAC, tuberculous lymphadenitis, reactive lymphadenitis.

INTRODUCTION: Cervical lymphadenopathy is a common clinical presentation in various departments of clinical practice.A high degree of sensitivity and specificity in diagnosing tuberculosis, malignancies by FNAC of lymph nodes has been noted in various studies ${ }^{1,2}$. FNAC has been successfully adopted as a preliminary line of investigation for diagnosis of cervical lymphadenopathy. Cervical lymph node surgical excisions are done only in cases inconclusive on cytology. In the present study we have analyzed the prevalence of various causes of lymphadenopathy in the local population with special reference to different age groups.

\section{AIMS AND OBJECTIVES:}

To know the overall prevalence of various causes responsible for cervical lymphadenopathy.

To know the distribution of various lesions among the age \& sex groups.

To emphasize the role of fine needle aspiration cytology (FNAC) in etiologic workup in significant cervical lymphadenopathy.

SUBJECT AND METHODS: Consecutive patients presented with cervical lymphadenopathyin our institute were recruited in thepresent study over the period of one year, from October 2011 to September 2012. Total 420 patients presented with cervical lymphadenopathy were assessed clinically, by laboratory and by FNAC study. Patients presenting with cervical lymphadenopathy of all age and sex group were included in the study.

All patients were asked detail history pertaining to neck swelling and relevant questions to the etiological factors. 
They underwent clinical examination of cervical lymphadenopathyi.e. size, shape, and consistency, matting etc. and extra cervical lymph nodes.

FNAC examination of the lymph node was done for diagnosis of pathological lesion. The palpable cervical node was fixed with one hand and the skin was cleansed and 22 gauze $-1.5 \mathrm{~cm}$ long needle with $10 \mathrm{ml}$ syringe was inserted into the lymph node and a full suction pressure was applied. The tip of the needle was moved around. The pressure was neutralized and the needle was withdrawn. The aspirated material was expelled on the glass slides. Four smears were made from each patient. Two slides were air dried and stained by Giemsa stain, while the remaining two slides were fixed in alcohol and then stained with haematoxylin and eosin stain for evaluation.

OBSERVATION: Out of 420, 18 patients were excluded from the study because of unsatisfactory aspirate of material from lymph nodes. Among remaining 402 patients, the age ranged from 6 months - 87yrs.33 cases (8.25\%) were in the age group of 0 -10yrs, $95(23.75 \%)$ cases in the age group of 11-20yrs, $105(26.25 \%)$ cases in the age group of 21-30 yrs., 63 (15.75\%) cases in the age group of 31-40yrs, $40(10 \%)$ cases in the age group of $41-50 \mathrm{yrs}, 23(5.7 \%)$ cases in the age group of 51-60 yrs., $22(5.5 \%)$ cases in the age group of $61-70$ yrs., $10(2.5 \%)$ cases were more than $70 y r s$.

215 cases were male (53.9\%) and 187 were female (46.1\%). FNAC was found to be simple, safe and convenient test without any record of complication.

Among the diagnostic outcome, (61)15.2\% were neoplastic lesions, (341) 84.8\% were nonneoplastic lesions.

Overall prevalence of various lesions, tuberculosis was the commonest 182 (45.5\%); followed by reactive lesion 109(27.2\%); Secondary metastases 47(11.7\%); Suppurative lymphadenitis 38(9.5\%); lymphoma 14(3.4\%); cystic lesion 8(1.9\%; (table 1)

Distribution of lesions in the various age groups. (Table 2).

33 cases(8.25\%) were in 1-10 yrs. age group, of them maximum were reactive lymphadenitis24(72.7\%), followed by tuberculosis 6 (18.8\%), Suppurative lymphadenitis2(6.0\%).

95 cases $(23.75 \%)$ were in 11-20yrs age group, of them reactive lymphadenitis were 35(36.8\%), tuberculosis 54(56.8\%), Suppurative lymphadenitis5(5.2\%), lymphoma 1(1.05\%).

105 cases $(26.25 \%)$ were in 21-30yrs age group, of them reactive lymphadenitis were 29 (27.6\%), tuberculosis 60 (57.1\%), Suppurative lymphadenitis 14 (13.3\%).

63 cases (15.75\%)were in 31-40 yrs. age group, of them reactive lymphadenitis were 11(17.4\%), tuberculosis 40(63.4.8\%), Suppurative lymphadenitis6(9.5\%), lymphoma 1(1.5\%), Metastatic deposits 5(7.9\%)

40 cases (10\%)were in 41-50 yrs. age group, of them reactive lymphadenitiswere $8(20 \%)$, tuberculosis 12(30\%), suppurative lymphadenitis6(15\%), lymphoma 2(5\%), metastatic deposits $12(30 \%)$.

$23(5.75 \%)$ cases in 51-60 yrs. age group, of them reactive lymphadenitis were $1(4.3 \%)$, tuberculosis 6(26\%), Suppurative lymphadenitis2(8.6\%), lymphoma 2(8.6\%), metastatic deposits $13(56.5 \%)$.

$22(5.5 \%)$ cases in 61-70 yrs. age group, reactive lymphadenitis were $1(4.5 \%)$ tuberculosis 4(18\%) Suppurative lymphadenitis1(4.5\%) lymphoma 4(18\%), metastatic deposits 13(59\%). 
$10(2.5 \%)$ cases in more than 70 yrs. age group, reactive lymphadenitis were $1(10 \%)$ tuberculosis 2(20\%) Suppurative lymphadenitis2(20\%) lymphoma 1(10\%), metastatic deposits $4(40 \%)$.

DISCUSSION:FNAC is a satisfactory tool in the diagnosis of tubercular and malignant lymphadenopathy. The sensitivity in diagnosing reactive and tubercular glands was nearly $90 \%$; the high specificity (98\% and $94.5 \%$ respectively) is regarded as an asset during bed-side evaluation in detecting pathological glands. The simplicity and cheapness of the procedure make it most suitable for use on outpatients basis even in peripheral hospitals and dispensaries. ${ }^{4}$ FNA of head and neck masses proved to be a useful tool in diagnosing metastasis with good certainty. ${ }^{1}$ The diagnosis offeredon FNAC has been shown to correlate very well with histopathological diagnosis after biopsy ${ }^{5}$

The incidence of cervical lymphadenopathy is more common in males than in females.

Maximum number of cases of cervical lymphadenopathy was noted in the age group of 11-40 yrs. comprising of $70 \%$ of the cases of this study with a peak incidence of $28.8 \%$ of cases in the $21-30$ yrs. age group.

The study documented the fact that out of 402 patients 61 patients $(15.2 \%)$ had neoplastic and 341 patients $(84.82 \%)$ had nonneoplastic lesions.

Among the various lesions the tuberculosis lesions were the most $n-1=182(45.5 \%)$ ranking on the top followed by reactive nonspecific chronic inflammation $n-2=109(27.2 \%)$ followed by metastases lesion n $3=47(11.75 \%)$ abscesses $n-4=38(9.5 \%)$ following by cysticlesion $n-$ $5=8(2.4 \%)$ and lastly the Lymphoma $n-6=14(3.4 \%)$

Reactive lymphadenitis was the most common cause of cervical lymphadenopathy in age group of 1-10yrs. Tuberculosis was the most common11 to 40 years. In 41 - 50 yrs. the incidence of tuberculosis and metastatic deposits were equal, after 50 years metastatic deposits was the most common.

Peak incidence of tuberculous cervical lymphadenopathy was noted in 11 to 40yrs, with lesser incidence in the younger (0-10yrs) and older age group after 40 yrs.

Peak incidence of reactive lymphadenitis was noted in the younger age group in 0-30 yrs., lesser incidence after 30 yrs., to almost nil after 50 yrs.

Peak incidence of metastatic disease was seen after 40 yrs., with a few cases in 30-40 yrs., nil before 30 yrs.

Peak incidence of Nonhodgkins lymphoma was seen in 61-70 yrs., lesser incidence in 31$60 \mathrm{yrs}$, and rare in less than 30 yrs.

Peak incidence of suppurative lymphadenitis was seen in 21-30 yrs. with a least incidence in very young and very old.

All cases of Hodgkin's lymphoma were noted in 25 -40yrs age group, and 75\% occurred in females.

Various studies have been done to analyze the prevalence of pathological lesions and etiological factors for cervical lymphadenopathy.

In the study by Janardhan etal6; conducted in north India in their series of 532 patients, performed FNAC of cervically mphadenopathy, reported $8.6 \%$ as malignant, 91.4 as benign. They noted a incidence of tuberculosis (51.9\%) and metastatic disease of (6.4\%). In the present study an increased incidence of malignant lesions (15.7\%) and metastatic disease (11.75\%) was noted. 
A study by Kim D.W et al2in a series of 1216 cases in Korea, of lymphadenopathies all over the body noted $73 \%$ benign lesions, $25.75 \%$ malignant lesions.

In a study by Rama Rao et al $^{7}$ of 2216 cases in south India of all superficial lymphadenopathy reported incidence of $37.7 \%$ of neoplastic lesions and $64.3 \%$ of non-neoplastic lesions.

In studies including lymphadenopathies in other parts of the body ${ }^{2}, 5$, the incidence of neoplastic lesions is higher (25.75\% and 37.7\%) when compared with the studies in cervical lymphadenopathy $(6.4 \%$ and $15.7 \%)$.In a study by Arun Roy et alin south India they found lymphomas to be the most commonest cause of lymphadenopathy in $44.5 \%$ of cases ${ }^{8}$.

Various FNAC studies of palpable supraclavicular lymph nodes revealed malignancy (metastatic deposits) the commonest etiology accounting 70-79.7\% of cases ${ }^{9}$.

In pediatric age group a study by $U$ Handa et al 10 , in north India they noted reactive lymphadenitis (62\%) as most common cause of cervical lymphadenopathy, followed by tuberculosis (25\%), neoplastic lesions in 1.3\%.

A study by Vamseedhar Annam et al ${ }^{11}$, of 324 cases in south India in pediatric age group noted reactive lymphadenitis in 58\%, tuberculosis in 30.5\%, neoplastic lesions in $5.6 \%$.

All the studies of cervical lymphadenopathy in pediatric age group 10,11 , including the present study suggest that reactive lymphadenitis is the commonest cause of cervical lymph node enlargement.

Reactive lymphadenitis is a rare a cause of cervical lymph node enlargement in older age group after 40yrs in the present study.

Infective etiology is the commonest cause of cervical lymphadenitis in the age group of 10-40 yrs., with peak incidence of tuberculous lymphadenitis and suppurative lymphadenitis in this age group.

In the present study Neoplastic lesions are the commonest cause of cervical lymphadenitis in the more than 40 age group with increased incidence of metastatic disease and non hodgkins lymphomas in this age group.

In a study by Huifand et al malignancy was more common among patients who were at least 50 yrs. old. Location of lymphadenopathy may be useful in predicting weather patient has cancer or benign disease and in their study $94 \%$ of patients with supraclavicular lymphadenopathy were diagnosed with cancer ${ }^{12}$.

All cases of Hodgkin's lymphomas were in the age group of 25 to 40 yrs. with $75 \%$ of cases affecting females.

The sensitivity rates of FNAC in tuberculosis, metastatic tumors, Hodgkin's disease, and nonHodgkin's lymphoma were found to be $83.3,97,30$, and $80.3 \%$, respectively, the specificity being $94.3,98.9,98.6$, and $95.4 \%$ in the same order. ${ }^{7}$ FNAC is a simple, inexpensive procedure, and when complemented by appropriate immuno cytochemical studies is accurate and reliable for routine diagnosis of lymphadenopathy.

In a study by Pandit $\mathrm{A}$ et al out of twenty cases of pyogenic lymphadenitis seven cases revealed tubercular lymphadenitis on subsequent histological examination ${ }^{13}$. 
Ultrasonography is useful in differentiating different causes of chronic cervical lymphadenitis in various studies, in adults and in pediatric age group. The ultrasonography helps in differentiating tuberculosis, lymphomas and metastasis ${ }^{14}$.

FNAC is an important diagnostic modality for the etiologic workup in significant cervical lymphadenopathy. It is almost as sensitive and specific as excision lymph node biopsy when an adequate aspirate is examined by expert eyes. ${ }^{11}$ FNAC also helps in the determination of the extent of the tumor, detection of recurrence, monitoring of the course of disease, obtaining of material for special studies such as microbiological cultures, immunological or genetic studies ${ }^{15}$.

CONCLUSION: The study concluded the fact that the tuberculosis is the most common cause of cervical lymphadenopathy in the south Asian population followed by reactive lymphadenitis and metastatic deposits.

Most cases of cervical lymphadenopathy occur in the age groups of 11-40yrs.

In pediatric age group, reactive lymphadenitis is the commonest cause of cervical lymph node enlargement.

Infective etiology is the commonest cause of cervical lymphadenitis in the age group of 11-40 yrs. with peak incidence of tuberculous lymphadenitis and suppurative lymphadenitis in this age group.

Neoplastic lesions are the commonest cause of cervical lymphadenitis in the more than 40 age group with increased incidence of metastatic disease and nonhodgkins lymphomas in this age group.

Reactive lymphadenitis is rarely a cause of cervical lymph node enlargement in older age group after 40yrs.

The incidence of neoplastic lesions is lesser in cervical lymphadenopathy when compared with the other lymph node groups.

Peak incidence of tuberculous cervical lymphadenopathy was noted in 11 to 40 yrs., with lesser incidence in the younger (0-10yrs) and older age group after $40 \mathrm{yrs}$.

\section{REFERENCES:}

1. I N Bagwan, S V Kane, R F Chinoy. Cytologic Evaluation of the Enlarged Neck Node-FNAC Utility in Metastatic Neck Disease. The Internet Journal of Pathology. 2007; 6: 2.

2. Kim DW, Jin SY, Lee DW, Lee CS. Diagnostic Usefulness of Fine Needle Aspiration Cytology on Lymphadenopathy-An analysis of 1, 216 Cases. Korean J Cytopathol 1997; 8(1):11-19.

3. Gadre DV, Singh UR, Saxena K, Bhatia A, Talwar V. Diagnosis of tubercular cervical lymphadenitis byFNAC microscopy and culture. IndianJ Tuberc 1991; 38:25.

4. Janardan V Bhatt, Jayashree M Shah, Falguni Shah. Clinico-Pathological profile of cervical lymphadenopathy. openmednic.in/1973/01/CLINICO; byChallenges

5. Rama Rao, Anil Prasad, Raghavan Narasimhan, Vanaja Sankaran. Fine-needle aspiration cytology in the diagnosis of superficial lymphadenopathyAn analysis of 2, 418 cases.Diagn Cytol 1998; 15: 382-6.

6. D. Malakar, ILN. Jajoo, Kiran Swarupet al. A Clinical evaluation of fine needle aspiration cytology in the diagnosis of lymphadenopathy.Indian J Tuberc 1991; 38:17. 
7. U Handa, $\mathrm{H}$ Mohanand A Bal. Role of fine needle aspiration cytology in evaluation of pediatric lymphadenopathy. Cytopathology 2003; 11:66 - 9.

8. Vamseedhar Annam, M H Kulkarni, Rekha B Puranik. Clinicopathologic Profile of Significant Cervical Lymphadenopathy in Children Aged 1. Actacytologica 2009; 153:174-8.

9. Harsh Kumar, Shirish S Chandanwale, Charusheela R Gore, Archana C Buch, Vijay H Satav, Pradhan M Pagaro. Role of fine needle aspiration cytology in assessment of cervical lymphadenopathy; Medical Journal of Dr.D.Y.Patil University 2013; 6:400-4.

10. Arun Roy, Rakhee Kar, Debdatta Basu, Bhavana Ashok Badhe. Spectrum of histopathologic diagnosis of lymph node biopsies:A descriptive study from a tertiary care centre in south India over 5 1/2 years. Indian J Pathol \& Microbial 2013; 56(2):103-8.

11. Abdulla A Abba, Mohammed Z Khalil. Clinical approach to lymphadenopathy. Annals of Nigerian medicine 2012; 6:11-17.

12. Hui Fand Lu, Xerxes Pundole, Shama Palla, Khanh Vu. Baseline characteristics predictive of malignancy amongpatients presenting with lymphadenopathy: ACancer center experience. Clinical Cancer Investigation Journal 2013; 12:325-9.

13. Pandey A, Kureel SN, Pandey J, Wakhlu A, Rawat J, Singh TB. Chronic cervical lymphadenopathy in children: Role of ultrasonography.J Indian Assoc Pediatr Surg 2012; 17:58-62.

14. Mitra S, Ray S, Mitra P K. Fine needle aspiration cytology of supraclavicular lymphnodes.Our experience over a three year period. J Cytol 2011; 28:108-10.

15. Pandit AA, Candes FP, Kubchandani SR. Fine needle aspiration cytology of lymph nodes. J postgrad med 1987; 33:134.

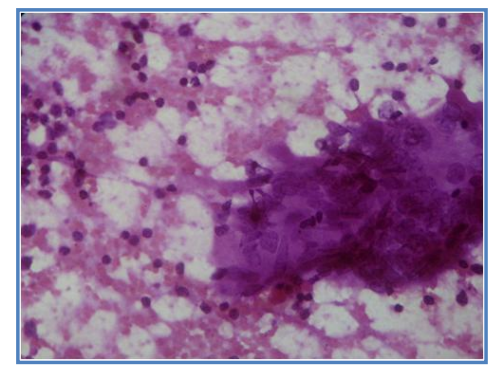

Fig. 1: Tuberculous lymphadenitis showing presence of

epithelioid granulomas in a background of lymphoid cells. H\&E, 10x.

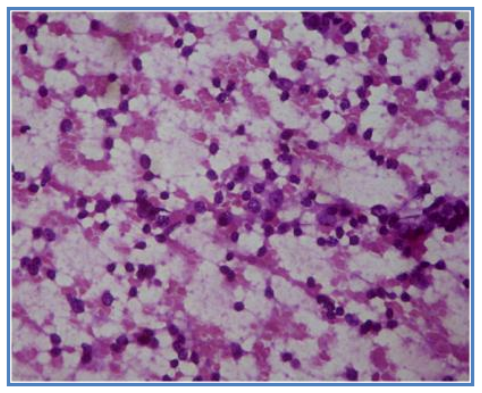

Fig. 2: Reactive lymphadenitis picture showing mixed population of lymphoid cells. H\&E, 10x. 


\section{ORIGINAL ARTICLE}

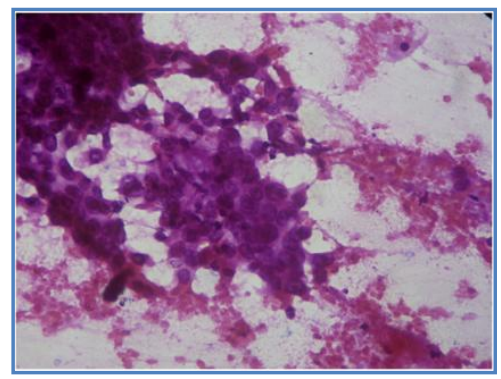

Fig. 3: Metastatic deposits shows presence of malignant cells with Hyperchromatic nucleus and nuclear pleomorphism. H\&E, 10x.

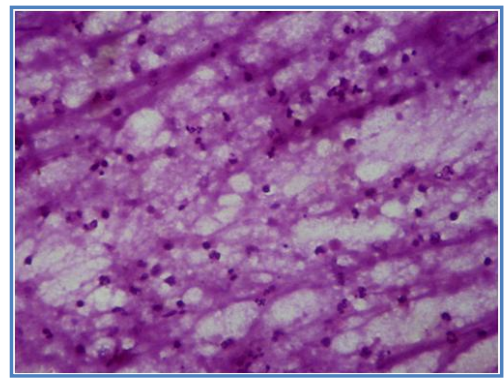

Fig. 4: Suppurative lymphadenitis showing a necrotic background and plenty of neutrophils. H\&E, 10x.

\begin{tabular}{|l|c|c|}
\hline \multicolumn{1}{|c|}{ DIAGNOSIS } & NUMBER OF CASES & PERCENTAGE \\
\hline TUBERCULOSIS & 182 CASES & $45.5 \%$ \\
\hline REACTIVE LYMPHADENITIS & 109 CASES & $27.2 \%$ \\
\hline SUPPURATIVE LYMPHADENITIS & 38 CASES & $9.5 \%$ \\
\hline METASTATIC DEPOSITS & 47 CASES & $11.75 \%$ \\
\hline NON HODGKINS LYMPHOMAS & 10 CASES & $2.5 \%$ \\
\hline HODGKINS LYMPHOMAS & 4 CASES & $1 \%$ \\
\hline \multicolumn{1}{|c|}{ TOTAL } & 402 CASES & $100 \%$ \\
\hline
\end{tabular}

TABLE- 1: INCIDENCE OF VARIOUS LESIONS IN CERVICAL LYMPHADENOPATHY

\section{AUTHORS:}

1. C. Nirmala

2. Dayananda. S. Biligi

3. Radha

\section{PARTICULARS OF CONTRIBUTORS:}

1. Associate Professor, Department of Pathology, Bangalore Medical College and RI, Bangalore.

2. Professor, Department of Pathology, Bangalore Medical College and RI, Bangalore.

3. Tutor, Department of Pathology, Bangalore Medical College and RI, Bangalore.

\section{NAME ADDRESS EMAIL ID OF THE} CORRESPONDING AUTHOR:

Dr. C. Nirmala,

No. $85,15^{\text {th }}$ Main,

Nanjundeshwara Layout,

J.P. Nagar, $5^{\text {th }}$ Phase, Bangalore -78 .

Email-chandrannirmala@yahoo.com

Date of Submission:01/12/2013.

Date of Peer Review: 02/12/2013.

Date of Acceptance: 19/12/2013.

Date of Publishing: 09/01/2014. 